\title{
The clinical indications and expected outcomes using bioabsorbable and non-bioabsorbable barriers in guided tissue regeneration therapies
}

\author{
Kevin George Murphy \\ Private Practice with Specialization in Periodontics, \\ Prosthodontics and Implant Dentistry in Baltimore
}

Guided Tissue Regeneration (GTR) is an accepted method of periodontal regeneration. However, widespread acceptance of this procedure is not seen due to variability in treatment outcome and the incidence of surgical complications. Biodegradable barriers are thought to enhance treatment outcomes by decreasing the incidence of complications. Evidence will be presented which addresses the cause of surgical complications, with emphasis being placed on flap management. This presentation will also discuss the healing patterns in surgical sites treated with biodegradable and non-biodegradable barriers. Guidelines for the indications for the use of each type of barrier will be presented. 